\title{
HÁBITOS ALIMENTARES DA COMUNIDADE IPG
}

\author{
FOOD HABITS OF THE IPG COMMUNITY \\ HÁBITOS ALIMENTARIOS DE LA COMUNIDAD IPG
}

\author{
Flávio Simão (falviosimao10@hotmail.com)* \\ José Alexandre Martins (jasvm@ipg.pt)** \\ Délia Simão (simao.delia@gmail.com)*** \\ Vítor Roque (vitor.roque@ipg.pt)****
}

\section{RESUMO}

Os hábitos alimentares possuem um papel fundamental na vida de um ser humano. O Instituto Politécnico da Guarda (IPG) possui um total de 2692 estudantes, que todos os dias fazem escolhas alimentares. Dada a sua importância, este estudo tem por objetivo avaliar: a) as escolhas preferências/seleções alimentares dos estudantes; b) o local do seu consumo e c) a sua composição corporal. Para tal, recorreu-se à realização de um inquérito por questionário, denominado "Hábitos Alimentares da Comunidade IPG", dirigido a todos os estudantes das quatro unidades orgânicas4 Responderam ao questionário cerca de 14\% dos estudantes do IPG. Após a análise dos resultados, conclui-se que seria importante sensibilizar os estudantes para a prática de uma alimentação saudável para tal deveriam ser realizadas várias ações de formação nas respetivas escolas, rastreios e até mesmo algumas competições de forma a que as boas escolhas alimentares se realizem de forma motivada e não obrigatória. Como os estudantes dão muita importância às cantinas, esta poderia ser um veículo importante para promover os bons hábitos alimentares.

Palavras-chave: Cantinas; Ensino Superior; Estudantes; Hábitos Alimentares; Índice de Massa Corporal.

\section{ABSTRACT}

Eating habits play a key role in human being's life. The Polytechnic Insti- 
tute of Guarda (IPG) has a total of 2692 students who, every day, make food choices and, given its importance, this study aims to evaluate the students' food choices, the place of their consumption, as well as their body composition. For this purpose, a survey, "Eating Habits of IPG Community", was addressed to all students of this Institute. The questionnaire was answered by almost 14\% of IPG students. After analysing the results, it was concluded that it would be important to sensitize the students to a healthy diet. Therefore, several training actions, namely, health screening and, even, some competitions should be carried out in every school, so that good food choices are made willingly and not in a compulsory way. As students consider canteens important, they could be important vehicles for promoting good eating habits.

Keywords: Body Mass Index; Canteens; Eating Habits; Higher Education; Students.

\section{RESUMEN}

Los hábitos alimentarios desempeñan un papel fundamental en la vida de un ser humano. El Instituto Politécnico da Guarda (IPG) tiene un total de 2692 estudiantes, que todos los días hacen elecciones alimentarias, y dada su importancia este estudio tiene por objetivo evaluar precisamente las elecciones alimentarias de los estudiantes, el lugar de su consumo así como su composición corporal. Para ello se ha recurrido a la realización de una encuesta denominada «Hábitos Alimentares da Comunidade IPG" a todos los estudiantes de las 4 escuelas del Instituto. Han contestado el cuestionario alrededor del 14\% del total de estudiantes del IPG. Después del análisis de los resultados se concluye que sería importante sensibilizar a los estudiantes para la práctica de una alimentación sana, para ello se cree importante la realización de varias acciones de formación en las respectivas escuelas, rastreos e incluso algunas competiciones de forma que las buenas elecciones alimentarias sean hechas de manera motivada y no obligatoria. Como los estudiantes dan mucha importancia a los comedores, esta podría ser un vehículo importante para promover los buenos hábitos alimentarios .

Palabras clave: Comedores; Enseñanza Superior; Estudiantes; Hábitos Alimentarios; Índice de Masa Corporal. 


\section{Egitania \\ $s$ c i e $n$ c i a}

* Estudante da Licenciatura em Gestão Hoteleira na Escola Superior de Turismo e Hotelaria do Instituto Politécnico da Guarda. Participante e segundo classificado da segunda edição do Portugal Future Hoteliers Summit.

** Professor Adjunto da Escola Superior Turismo e Hotelaria (ESTH) do Instituto Politécnico da Guarda (IPG), Portugal. Membro da UTC de Ciências Exatas e Experimentais do IPG. Membro da Unidade de Investigação para o Desenvolvimento do Interior (UDI/ IPG).

*** Licenciada em Ciências da Nutrição pela Faculdade de Ciências da Nutrição e Alimentação da Universidade do Porto. Membro efetivo da ordem dos nutricionistas (N1458). Colaborou no Inquérito Alimentar Nacional e de Atividade Física da Universidade do Porto.

**** Professor Adjunto da Escola Superior de Turismo e Hotelaria (ESTH) do Instituto Politécnico da Guarda (IPG), Portugal. Membro da UTC de Turismo e Hotelaria do IPG. Membro da Unidade de Investigação para o Desenvolvimento do Interior (UDI/IPG). 


\section{INTRODUÇÃO}

A alimentação possui um conjunto de funções como, por exemplo, assegurar a sobrevivência do Homem, fornecer nutrientes e energia necessária ao bom funcionamento do organismo, contribuir para a manutenção do nosso estado de saúde físico e mental, prevenir certas doenças tais como obesidade, diabetes, doenças cardiovasculares entre outras e contribuir para o adequado crescimento e desenvolvimento das crianças e adolescentes (Mahan, Escott-Stump, \& Raymond, 2013).

O consumo alimentar, ao longo dos anos, tem vindo a alterar-se devido ao desenvolvimento económico e ao aumento do poder de compra, que determinou um aumento na disponibilidade alimentar e na acessibilidade aos serviços (Moreira \& Padrão, 2004).

Os hábitos alimentares são definidos como "tipos de escolha e consumo de alimentos por um indivíduo, ou grupo, em resposta a influências fisiológicas, psicológicas, culturais e sociais" (Dutra-de-Oliveira \& Marchini, 2008). De acordo com a European Food Information Council (EuFIC) a escolha dos hábitos alimentares deriva de fatores vários tais como: biológicos, económicos, físicos, sociais, psicológicos, regionais e culturais (Eufic, 2006).

Como os hábitos alimentares têm um papel fundamental na vida de um ser humano e como estes interferem no desenvolvimento cognitivo de cada individuo, considerou-se que seria interessante perceber que hábitos alimentares têm os estudantes que pertencem à comunidade IPG. O principal objetivo desta investigação é o de avaliar os comportamentos alimentares dos estudantes e perceber se existem diferenças entre os estudantes que pertencem às diferentes escolas do IPG. $O$ IPG é constituído por 4 escolas nomeadamente: a Escola Superior de Turismo e Hotelaria (ESTH), a Escola Superior de Saúde (ESS), a Escola Superior de Educação, Comunicação e Desporto (ESECD) e a Escola Superior de Tecnologia e Gestão (ESTG). À exceção da ESTH, que se localiza na cidade de Seia, as restantes escolas estão todas localizadas na cidade da Guarda.

Estudos como o de Davy, Benes, \& Driskell (2006) e Deshmukh-Taskar, Nicklas, Yang, \& Berenson (2007) revelam que os principais determinantes das escolhas alimentares realizadas pelos jovens universitários são a falta de tempo disponível, a conveniência, o custo, o sabor, o 
estado de saúde, o ambiente social e físico bem como as preocupações com o controlo de peso corporal.

Julgou-se também que seria importante avaliar como é que os estudantes se encontram na relação do peso com a altura, uma vez que este indicador representa bem os hábitos alimentares de um indivíduo.

Outro dos objetivos é perceber a importância da cantina para os estudantes, se estes gostam ou não da oferta que lhes é posta à disposição diariamente e perceber se existem diferenças significativas entre as opiniões dos estudantes das diferentes escolas.

Paralelamente considerou-se também que seria interessante perceber como é o comportamento dos estudantes em termos do consumo de fast-food e que diferenças existem a esse nível entre os estudantes das várias escolas.

\section{METODOLOGIA}

O presente trabalho caracteriza alguns dos hábitos alimentares dos estudantes que atualmente frequentam o IPG nos vários níveis de formação do ensino superior politécnico, nomeadamente em Cursos de Especialização Tecnológica, Licenciaturas, Pós-graduações e Mestrados, distribuídos pelas quatro escolas que fazem parte desta instituição de ensino superior.

Para conseguir obter a informação necessária e uma conclusão mais precisa sobre aqueles que são alguns dos hábitos alimentares atuais destes estudantes, foi desenvolvido um questionário que foi implementado online na plataforma Google Docs. O questionário foi dividido em três partes: a primeira parte é referente aos dados sociodemográficos do aluno e constituída por 9 questões, a segunda parte apresenta questões relacionadas com os hábitos alimentares de cada um dos estudantes e constituída por 16 questões e a terceira e última parte é referente às cantinas do IPG e constituída por 3 questões, o que perfaz um total de 28 questões.

O link para o referido questionário esteve disponível na plataforma de e-Learning do IPG - Blackboard, por se considerar ser a forma mais eficaz e eficiente de se conseguir que toda a população do estudo tivesse acesso ao mesmo. Para tal foram enviados e-mails para as Direções das quatro escolas que formam o IPG, para que estas também 
incentivassem os estudantes a preencher o questionário.

A recolha de dados ocorreu entre 27 de novembro de 2014 e 15 de fevereiro de 2015. A população deste estudo foi o total de estudantes matriculados nas quatro escolas do IPG, ou seja 2692 estudantes, de acordo com os dados recolhidos junto dos Serviços Académicos do IPG, a 31 de outubro de 2014.

No total foram contabilizados 387 questionários, dos quais 5 foram eliminados por ser notória a falta de coerência nas respostas dadas, designadamente na questão relativamente ao peso. Assim, foram validados 382 questionários, cerca de 14\% da população, sendo esta a dimensão da amostra do estudo.

Posteriormente os dados foram tratados na aplicação Microsoft Excel e na aplicação IBM SPSS Statistics (versão 24.0), sendo determinadas as frequências, algumas estatísticas descritivas, bem como o cruzamento de algumas variáveis para uma melhor perceção da realidade.

Relativamente às variáveis altura e peso, decidiu-se seguir a mesma abordagem de classificação dos dados por classes que foi seguida para as idades. Com base na dimensão da amostra e seguindo a regra de Struges (Pestana \& Gageiro, 2014), estabeleceu-se o número de classes que deveria ser usado para agrupar as respetivas alturas e pesos, bem como a amplitude de cada classe. Além disso, com base nestas duas variáveis calculou-se o Índice de Massa Corporal (IMC) pois pensou-se que seria interessante perceber como é que varia o IMC dos estudantes do IPG em função do género. O cálculo do IMC (OMS, n.d.) de cada aluno foi realizado a partir do peso e da altura relatados, através da fórmula: . Os pontos de corte utilizados para a categorização do IMC foram os preconizados pela Organização Mundial de Saúde (OMS) (OMS, n.d.):

- $\quad<18,5$ - Magreza

- 18,5-24,99 - Peso normal

- 25,0-29,99 - Pré-Obesidade

- 30,0-34,99 - Obesidade grau 1

- 35,0-39,99 - Obesidade grau II

- $\quad \geq 40$ - Obesidade grau III ou Mórbida

Associado ainda aos hábitos de alimentação saudável, observou-se 
os dados dos estudantes relativos ao comer fast-food e à escola que frequentam, de forma a perceber se existe alguma relação entre essas variáveis.

Por fim, considerou-se útil analisar se existem diferenças significativas entre escolas no que diz respeito à adesão às cantinas disponíveis.

\section{RESULTADOS}

Responderam ao questionário "Hábitos Alimentares da Comunidade IPG" 382 estudantes, ou seja, cerca de 14\% do total de estudantes do IPG. Nesta amostra 250 eram mulheres, o que corresponde a 65\% do total dos inquiridos. Esta percentagem não coincide totalmente com a distribuição por género dos estudantes matriculados no IPG no ano letivo 2014/2015 que foi de 52,3\% de mulheres. Dos respondentes 86 (22,5\%) são estudantes provenientes da ESECD, 102 (26,7\%) da ESTG, 67 (17,5\%) da ESTH e 107 (28\%) da ESS.

Com os dados obtidos, podemos concluir que a média da idade das mulheres que responderam ao inquérito é de 22,6 ( $\pm 6,42)$ anos, a média das alturas corresponde a 1,6 $\pm 0,06 \mathrm{~m}$ e a média referente ao peso corresponde a 59,3 $\pm 9,48 \mathrm{Kg}$.

Relativamente aos homens podemos concluir que a média das idades foi de 24,9 98,00 anos. Estes homens têm uma altura média que corresponde a 1,77 $\pm 0,07 \mathrm{~m}$. A média do peso dos homens corresponde a um total de $74 \pm 10,30 \mathrm{~kg}$, o que está em consonância com o facto da média das alturas ser superior.

Através dos dados referentes ao peso e à altura dos inquiridos calculouse o IMC (Tabela 1 e Gráfico 1).

A maioria dos inquiridos ( $N=285 ; 75 \%)$ situa-se na categoria de peso normal, contudo 84 (22\%) estudantes apresentam sinais de pré-obesidade ou obesidade grau I, não havendo estudantes nas categorias de obesidade grau II e III. Por outro lado, há um conjunto de 13 (3\%) de estudantes que apresenta sinais de magreza. 


\begin{tabular}{|l|c|c|c|c|c|c|}
\multicolumn{2}{c}{ IMC } & \multicolumn{2}{c}{ Homems } & \multicolumn{2}{c}{ Mulheres } & \multicolumn{2}{c|}{ Total } \\
\cline { 2 - 7 } \multicolumn{1}{c|}{} & $\mathrm{N}$ & $\%$ & $\mathrm{~N}$ & $\%$ & $\mathrm{~N}$ & $\%$ \\
\hline$<18,5$ & 10 & $4 \%$ & 3 & $2 \%$ & 13 & $3 \%$ \\
\hline $18,5-24,99$ & 202 & $81 \%$ & 83 & $63 \%$ & 285 & $75 \%$ \\
\hline $25-29,99$ & 27 & $11 \%$ & 37 & $28 \%$ & 64 & $17 \%$ \\
\hline $30-34,99$ & 11 & $4 \%$ & 9 & $7 \%$ & 20 & $5 \%$ \\
\hline $35-39,99$ & 0 & $0 \%$ & 0 & $0 \%$ & 0 & $0 \%$ \\
\hline$>=40$ & 0 & $0 \%$ & 0 & $0 \%$ & 0 & $0 \%$ \\
\hline
\end{tabular}

Tabela 1 - IMC por categoria.

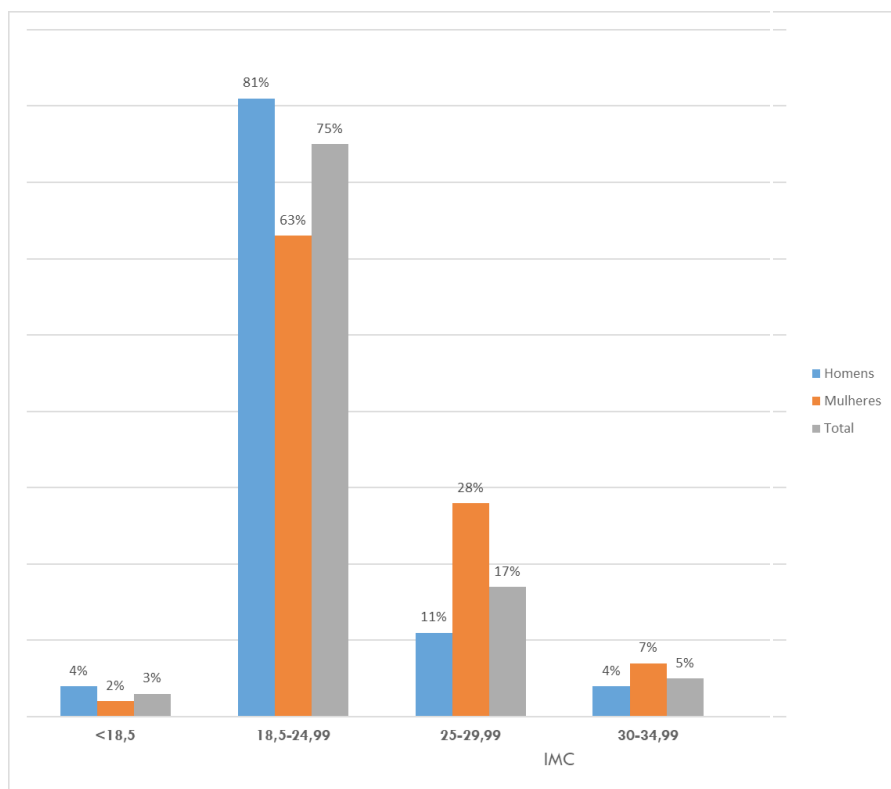

Gráfico 2 - IMC por categoria.

Cerca de 65\% da amostra, fazem entre quatro a cinco refeições por dia, o que nos leva a extrapolar que correspondem ao pequeno-almoço, almoço, lanche, jantar e ceia. Cerca de 4\% dos estudantes respondentes só fazem em média duas refeições diariamente e apenas 2\% fazem mais de 6 refeições por dia.

A maioria destes estudantes (74\%) fazem as suas refeições em casa, sendo que apenas uma minoria (2\%) diz fazer habitualmente as suas 
refeições semanais em casa de amigos, e apenas 1\% aponta a casa de familiares como opção.

No que diz respeito ao pequeno-almoço, 86\% dos estudantes respondentes tomam o pequeno-almoço, sendo que apenas 3\% respondeu que não e 12\% respondeu que raramente tomava o pequeno-almoço. O que é mais apontado como sendo consumido ao pequeno-almoço é o café, o leite, pão e torradas. O local apontado com maior percentagem para a realização desta refeição é "em casa", com cerca de 87\%, e ainda $2 \%$ responde que costuma realizar esta refeição num café/pastelaria, sendo que os outros 11\% apontaram diversos outros sítios, entre os quais o próprio bar da escola.

Da nossa amostra podemos afirmar que cerca de 68\% consome entre uma a duas peças de fruta por dia, 20\% responderam que não comem fruta e 12\% diz que come três ou mais peças de fruta por dia.

Relativamente ao consumo semanal de peixe, cerca de $73 \%$ dos inquiridos responderam que comem menos de 3 vezes peixe por semana, $6 \%$ simplesmente não come peixe e ainda existe $22 \%$ da amostra que respondeu que come peixe mais de 3 vezes por semana.

No que respeita ao consumo semanal de salada e legumes, com a mesma percentagem, 37\%, surgem os que consomem menos de 3 vezes por semana e os que consomem mais de 3 vezes por semana, $17 \%$ responderam que ingerem estes alimentos todos os dias e apenas 9\% não consomem nenhum destes alimentos por semana.

O consumo de sopa é muito semelhante ao consumo de saladas e legumes, sendo que a percentagem dos que ingere menos de 3 vezes por semana e a percentagem dos que ingerem mais de 3 vezes por semana é respetivamente 33\% e 35\%. Além disso, 28\% diz que ingere sopa todos os dias e 8\% não ingere sopa.

Metade da nossa amostra ingere entre 0,5 e 1 litro de água por dia, sendo que apenas $6 \%$ ingere o consumo de água diário recomendado pelos especialistas, ou seja, mais de 1,5 I por dia (Mahan et al., 2013). No que diz respeito ao consumo de leite e derivados, 93\% dos inquiridos afirmam consumir este tipo de alimentos, sendo que os restantes (7\%) afirmam não os consumir.

Em relação às cantinas, 319 estudantes, cerca de 84\% da amostra, refere que gosta de comer na cantina. Relativamente aos estudantes de cada escola que responderam que gostam da comida da cantina, a 
escola que apresenta uma maior percentagem é a ESECD com cerca de 87\%. A escola que apresenta menor percentagem, embora com uma diferença pouco significativa, é a ESTH com cerca de 81\% (Tabela 2)

\begin{tabular}{|c|c|c|c|c|c|}
\hline & & & Não & Sim & Total \\
\hline \multirow[t]{12}{*}{ Escolas } & & Contagem & 11 & 75 & 86 \\
\hline & \multirow[t]{3}{*}{ ESECD } & $\%$ em Escolas & $12,80 \%$ & $87,20 \%$ & $100,00 \%$ \\
\hline & & $\%$ do Total & $2,90 \%$ & $19,60 \%$ & $22,50 \%$ \\
\hline & & Contagem & 19 & 88 & 107 \\
\hline & \multirow[t]{3}{*}{ ESS } & $\%$ em Escolas & $17,80 \%$ & $82,20 \%$ & $100,00 \%$ \\
\hline & & $\%$ do Total & $5,00 \%$ & $23,00 \%$ & $28,00 \%$ \\
\hline & & Contagem & 20 & 102 & 122 \\
\hline & \multirow[t]{3}{*}{ ESTG } & $\%$ em Escolas & $16,40 \%$ & $83,60 \%$ & $100,00 \%$ \\
\hline & & $\%$ do Total & $5,20 \%$ & $26,70 \%$ & $31,90 \%$ \\
\hline & & Contagem & 13 & 54 & 67 \\
\hline & \multirow[t]{2}{*}{ ESTH } & $\%$ em Escolas & $19,40 \%$ & $80,60 \%$ & $100,00 \%$ \\
\hline & & $\%$ do Total & $3,40 \%$ & $14,10 \%$ & $17,50 \%$ \\
\hline \multirow{2}{*}{ Total } & & Contagem & 63 & 319 & 382 \\
\hline & & $\%$ do Total & $16,50 \%$ & $83,50 \%$ & $100,00 \%$ \\
\hline
\end{tabular}

Tabela 3 - Adesão às cantinas.

No entanto as percentagens associadas a cada escola individualmente estão bastante próximas umas das outras, sendo que a diferença entre a que reúne maior anuência, ESECD, e a que reúne menor anuência, ESTH, é de apenas 6\%. Além disso, de acordo com a tabela de contingência e o valor $V$ de Cramer, que apresentou uma significância aproximada de 0,707>0,05, pode concluir-se que não há diferenças estatísticas significativas quanto ao gosto pela comida da cantina entre as várias escolas. Assim, parece que a maioria dos estudantes estão satisfeitos com a comida que é servida nas cantinas do IPG e que não há diferenças a este nível entre os alunos das várias escolas do IPG.

Os estudantes ainda foram questionados se achavam que seria conveniente que a cantina tivesse serviço de pequeno-almoço e lanche, questão essa que obteve 68\% de respostas afirmativas, o que vem reforçar a importância que a cantina tem para os estudantes. 
Relativamente ao consumo de fast-food, os dados obtidos nos questionários mostram que a percentagem total dos estudantes inquiridos que não comem fast-food é de 5\%, ou seja, os outros 95\% da nossa amostra comem fast-food, pelo menos uma vez por mês. Das quatro escolas inquiridas a que apresenta maior percentagem de respondentes que comem menos fast-food é a ESECD, correspondendo a 8\% do total de inquiridos referentes a essa escola e representando 1,8\% do total da nossa amostra (Tabela 3). Por outro lado, a escola que apresenta menor percentagem (3\%) de respondentes que comem menos fast-food é a ESS, o que equivale a 0,8\% da amostra (Tabela 3).

\begin{tabular}{|c|c|c|c|c|}
\hline \multirow[b]{2}{*}{ Escola } & \multirow{2}{*}{$\begin{array}{c}N^{\circ} \text { de respostas } \\
\text { por escola }\end{array}$} & \multicolumn{3}{|c|}{ Não comem fast-food } \\
\hline & & $\mathrm{N}$ & $\%$ Escola & $\%$ Total \\
\hline ESECD & 86 & 7 & $8 \%$ & $1,80 \%$ \\
\hline ESS & 107 & 3 & $3 \%$ & $0,80 \%$ \\
\hline ESTG & 122 & 7 & $6 \%$ & $1,80 \%$ \\
\hline ESTH & 67 & 4 & $6 \%$ & $1,00 \%$ \\
\hline Total & 382 & & & $5 \%$ \\
\hline
\end{tabular}

Tabela 4 - Não consumo de fast-food.

Paralelamente a estes dados, o consumo de comida pré-cozinhada não é muito utilizado pelos estudantes da comunidade do IPG, sendo que responderam que raramente consumiam este tipo de comida cerca de $68 \%$. Responderam que consumiam este tipo de comida 24\%, que nunca comiam este tipo de comida $7 \%$ e, ainda, $2 \%$ respondeu que consome este tipo de comida muitas vezes.

\section{CONCLUSÃO}

Com a investigação realizada para o desenvolvimento deste trabalho pode-se percecionar que os estudantes do IPG não apresentam os melhores hábitos alimentares, sendo esta ideia reforçada pelas altas percentagens dos estudantes que consomem fast-food com alguma 
frequência, tal como estudado por Papadaki, Hondros, A. Scott, \& Kapsokefalou (2007) em estudantes universitários gregos. Ainda neste último estudo referido, os estudantes gregos que vivem fora de casa diminuíram o consumo semanal de frutas frescas, vegetais e peixe, e aumentaram o consumo de açúcar vinho, álcool e fast-food.

Para superar algumas das lacunas nos hábitos alimentares encontradas neste estudo, os estudantes deveriam incluir uma maior variedade de alimentos nas suas refeições, nomeadamente: comendo mais sopas, uma vez que existe cerca de $41 \%$ dos estudantes a comer sopa menos de 3 vezes por semana; comendo mais vegetais e legumes, já que existem cerca de $41 \%$ dos estudantes respondentes a afirmar que consomem estes alimentos menos de 3 vezes por semana; elevando o consumo semanal de peixe, sendo que existe cerca de $79 \%$ dos respondentes a responder que consomem peixe menos de 3 vezes por semana; e comendo pelo menos três peças de fruta por dia, que é o que recomendam as regras de uma boa alimentação (Mahan et al., 2013), sendo que 87\% da amostra afirma que come menos de 3 peças de fruta por dia.

Em relação à avaliação do IMC, encontraram-se percentagens consideráveis de estudantes com peso acima do normal ou obesidade grau I, dentro dos quais as mulheres se destacam ainda mais, o que vem de encontro ao estudo feito à população portuguesa designado "Inquérito Alimentar Nacional e de Atividade Física" (Lopes et al., 2017), que concluiu que $22,3 \%$ dos inquiridos apresentavam obesidade, sendo a maior percentagem nas mulheres (24,3\% vs 20,1\%).

Para ações futuras, conclui-se que seria importante sensibilizar os estudantes para este tipo de prática com o objetivo de os levar a ter mais cuidado com a alimentação. Por isso deveriam ser feitas ações de formação nas diferentes escolas, rastreios e até mesmo algumas competições que possam levar à prática de bons hábitos alimentares para que comer bem seja uma motivação e não uma obrigação. Dada a importância que os estudantes atribuem à cantina, esta poderia ser um veículo importante para promover os bons hábitos alimentares.

Este estudo possui como limitação a amostra, que representa cerca de 14\% dos estudantes da comunidade IPG, sendo que assim se torna difícil perceber quais são os verdadeiros hábitos alimentares dos estudantes, uma que vez os inquiridos são uma minoria. No entanto, acre- 
ditamos que os valores que foram apresentados não fogem muito da atual realidade geral dos hábitos alimentares destes estudantes.

\section{BIBLIOGRAFIA}

Davy, S. R., Benes, B. A., \& Driskell, J. A. (2006). Sex Differences in Dieting Trends, Eating Habits, and Nutrition Beliefs of a Group of Midwestern College Students. Journal of the American Dietetic Association, 106(10), 1673-1677. https://doi.org/10.1016/i.jada.2006.07.017

Deshmukh-Taskar, P., Nicklas, T. A., Yang, S.-J., \& Berenson, G. S. (2007). Does Food Group Consumption Vary by Differences in Socioeconomic, Demographic, and Lifestyle Factors in Young Adults? The Bogalusa Heart Study. Journal of the Academy of Nutrition and Dietetics, 107(2), 223-234. https://doi. org/10.1016/i.jada.2006.11.004

Dutra-de-Oliveira, J. E., \& Marchini, J. S. (2008). Ciências Nutricionais - Aprendendo a Aprender (2nd ed.). Sarvier.

Eufic. (2006). The determinants of food choice.

Lopes, C., Torres, D., Oliveira, A., Severo, M., Alarcão, V., Guiomar, S., ... Ramos, E. (2017). Inquérito Alimentar Nacional e de Atividade Física IAN-AF 2015 2016 - parte II.

Mahan, L. K., Escott-Stump, S., \& Raymond, J. L. (2013). Krause - Alimentos, Nutrição e Dietoterapia (13th ed.). Elsevier.

Moreira, P. A., \& Padrão, P. D. (2004). Educational and economic determinants of food intake in Portuguese adults: a cross-sectional survey. BMC Public Health, 4(1), 58. https://doi.org/10.1 186/1471-2458-4-58

OMS. (n.d.). WHO :: Global Database on Body Mass Index. Retrieved July 3, 2017, from http://www.euro.who.int/en/health-topics/disease-prevention/nutrition/a-healthy-lifestyle/body-mass-index-bmi

Papadaki, A., Hondros, G., A. Scott, J., \& Kapsokefalou, M. (2007). Eating habits of University students living at, or away from home in Greece. Appetite, 49(1), 169-176. https://doi.org/10.1016/i.appet.2007.01.008

Pestana, M. H., \& Gageiro, J. N. (2014). Análise de Dados para Ciências Sociais (6th ed.). Edições Sílabo. 